\title{
Intraductal papillary neoplasms of the bile duct: stepwise progression to carcinoma involves common molecular pathways
}

\author{
Anna Melissa Schlitter ${ }^{1}$, Diana Born ${ }^{2}$, Marcus Bettstetter ${ }^{3}$, Katja Specht ${ }^{1}$, \\ Corina Kim-Fuchs ${ }^{4}$, Marc-Oliver Riener ${ }^{5}$, Petia Jeliazkova ${ }^{6}$, Bence Sipos ${ }^{7}$, Jens T Siveke $^{6}$, \\ Benoit Terris $^{8}$, Yoh Zen ${ }^{9}$, Tibor Schuster ${ }^{10}$, Heinz Höfler ${ }^{1}$, Aurel Perren ${ }^{2}$, Günter Klöppel ${ }^{1}$ \\ and Irene Esposito ${ }^{1}$
}

${ }^{1}$ Institute of Pathology, Technische Universität München, München, Germany; ${ }^{2}$ Institute of Pathology, University of Bern, Bern, Switzerland; ${ }^{3}$ Molecular Pathology South Bavaria, Trogerstr. 18, München, Germany; ${ }^{4}$ Department of Visceral Surgery and Medicine, Inselspital, Bern, Switzerland; ${ }^{5}$ Institute of Pathology, University Hospital Erlangen, Erlangen, Germany; ${ }^{6}$ Department of Internal Medicine, Klinikum rechts der Isar, Technische Universität München, München, Germany; ${ }^{7}$ Institute of Pathology, University of Tübingen, Tübingen, Germany; ${ }^{8}$ Division of Pathological Anatomy, Hôpital Cochin, Université Paris Descartes, Paris, France; ${ }^{9}$ Institute of Liver Studies, King's College Hospital, London, UK and ${ }^{10}$ Department of Medical Statistics and Epidemiology, Technische Universität München, München, Germany

Intraductal papillary neoplasms of the bile duct are still poorly characterized regarding (1) their molecular alterations during the development to invasive carcinomas, (2) their subtype stratification and (3) their biological behavior. We performed a multicenter study that analyzed these issues in a large European cohort. Intraductal papillary neoplasms of the bile duct from $\mathbf{4 5}$ patients were graded and subtyped using mucin markers and CDX2. In addition, tumors were analyzed for common oncogenic pathways, and the findings were correlated with subtype and grade. Data were compared with those from 22 extra- and intrahepatic cholangiocarcinomas. Intraductal papillary neoplasms showed a development from preinvasive low- to highgrade intraepithelial neoplasia to invasive carcinoma. Molecular and immunohistochemical analysis revealed mutated KRAS, overexpression of TP53 and loss of $p 16$ in low-grade intraepithelial neoplasia, whereas loss of SMAD4 was found in late phases of tumor development. Alterations of HER2, EGFR, $\beta$-catenin and GNAS were rare events. Among the subtypes, pancreato-biliary $(36 \%)$ and intestinal $(29 \%)$ were the most common, followed by gastric $(18 \%)$ and oncocytic $(13 \%)$ subtypes. Patients with intraductal papillary neoplasm of the bile duct showed a slightly better overall survival than patients with cholangiocarcinoma (hazard ratio (cholangiocarcinoma versus intraductal papillary neoplasm of the bile duct): 1.40 ; $95 \%$ confidence interval: $0.46-4.30$; $\boldsymbol{P}=\mathbf{0 . 5 5 2}$ ). The development of biliary intraductal papillary neoplasms of the bile duct follows an adenomacarcinoma sequence that correlates with the stepwise activation of common oncogenic pathways. Further large trials are needed to investigate and verify the finding of a better prognosis of intraductal papillary neoplasms compared with conventional cholangiocarcinoma.

Modern Pathology (2014) 27, 73-86; doi:10.1038/modpathol.2013.112; published online 5 July 2013

Keywords: bile duct tumors; GNAS; intraductal precursor lesion; KRAS

Bile duct carcinoma is a rare disease with an extremely poor prognosis. As most tumors are unresectable at the time of diagnosis, early detection at a preinvasive stage is the only hope of cure. Two

Correspondence: Professor I Esposito, MD, Institute of Pathology, Technische Universität München, Ismaningerstr. 22, Munich 81675, Germany.

E-mail: esposito@lrz.tum.de

Received 14 February 2013; revised 6 May 2013; accepted 11 May 2013; published online 5 July 2013 entities are considered to be precursor lesions of cholangiocarcinoma: biliary intraepithelial neoplasia $^{1}$ and intraductal papillary neoplasms. ${ }^{2}$ Intraductal papillary neoplasms of the bile duct have been recently included in the World Health Organisation classification of bile duct tumors ${ }^{3,4}$ and comprise the previous categories of biliary papilloma and papillomatosis. Intraductal papillary neoplasms of the bile duct are considered to be the biliary counterpart of pancreatic intraductal papillary mucinous neoplasms. ${ }^{2,5}$ Concerning pancreatic 
intraductal papillary mucinous neoplasm, the existence of four different subtypes (intestinal, gastric, pancreato-biliary and oncocytic) is well established, ${ }^{6}$ and prognostic differences among subtypes have been described. ${ }^{7-10}$ In addition, several studies performed on human and transgenic mice revealed a number of genetic changes in key molecular pathways during intraductal papillary mucinous neoplasm development. ${ }^{11-16}$

In contrast to the more common pancreatic intraductal papillary mucinous neoplasms, intraductal papillary neoplasms of the bile duct have not been well characterized so far, either from the clinical or from the molecular point of view. Most studies of intraductal papillary neoplasms of the bile duct are based on small number of patients,,$^{2,17-21}$ and the three largest studies (55-97 patients) ${ }^{22-24}$ exclusively enrolled Asiatic patients, a considerable proportion of them with hepatolithiasis or Clonorchiasis infection. ${ }^{24}$ Therefore, little is known about the prognostic relevance of the histological subtypes ${ }^{17,23}$ and the oncogenic pathways underlying the development of these tumors, especially of those occurring sporadically in Western countries. ${ }^{25-29}$

In this study, we analyzed a large series of intraductal papillary neoplasms of the bile duct from the patients of European origin, with focus on molecular genetic changes in relation to morphology, distribution of different subtypes and their prognostic relevance. The data were then correlated with the biology of the tumors.

\section{Materials and methods}

\section{Patient Selection and Follow Up}

Extrahepatic bile duct and liver tumor specimens from patients treated in various European institutions and fulfilling the diagnostic criteria of intraductal papillary neoplasm of the bile duct (ie, neoplasms of the intra- or extrahepatic bile ducts with prominent intraductal papillary growth) were included in this study. Papillary neoplasms of the ampulla, biliary intraductal tubulo-papillary neoplasms, biliary mucinous cystic neoplasms and biliary intraepithelial neoplasia were excluded. The study cohort consisted of 45 patients from six different institutions: 13 cases came from the Institute of Pathology and 6 cases from the Institute's consultation files, Technische Universität München, München, Germany; 11 cases from the Institute of Pathology, University of Bern, Bern, Switzerland; 6 cases from the Institute of Pathology, University of Tübingen, Tübingen, Germany; 4 cases from the Pathology Division of Pathological Anatomy, Hôpital Cochin, Université Paris Descartes, Paris, France; 3 cases from the Institute of Liver Studies, King's College Hospital, London, UK; and 2 cases from the Institute of Pathology, University Hospital of Erlangen, Erlangen, Germany.
One of the cases was previously published as case report. ${ }^{30}$ Twenty-two patients with invasive intra- or extrahepatic cholangiocarcinoma without any intraductal papillary components and therefore possibly originating from biliary intraepithelial neoplasia lesions served for comparison. Clinical, demographic and macroscopic information was obtained by review of the medical charts and pathology reports. The histopathological diagnosis and grading, as well as staging, followed the recommendations of the World Health Organisation. ${ }^{3,4}$ The study was approved by the ethics committee of the Technische Universität München, München, Germany. Patients were followed after resection or biopsy until death or last follow-up in March 2012. Patients with perioperative death within the first 30 days (three patients with intraductal papillary neoplasm of the bile duct (8\%, 95\% confidence interval: $2-22 \%$ ) and four cholangiocarcinoma patients $(20 \%, 95 \%$ confidence interval: $6-44 \%$ )) were excluded from survival analysis.

\section{Histology and Immunohistochemistry}

All specimens were fixed in $4 \%$ formaldehyde, embedded in paraffin, cut in 3-5 micrometer-thick sections and stained with hematoxylin and eosin. Histological and immunohistochemical analyses were performed on representative tissue blocks (one to maximum three blocks, average 1.2) for the intraductal papillary neoplasm of the bile duct cases and on a tissue microarray (two 2-mm tissue cores/sample) for the cholangiocarcinoma cases. The following antibodies were used: anti-MUC1, anti-MUC6 (Novocastra, Leica Biosystems Newcastle, Newcastleupon-Tyne, UK; 1:50), anti-EGFR, antiTP53, anti-HER2 (DakoCytomation, Glostrup, Denmark; 1:50, 1:300 and 1:300, respectively), anti-MUC2, anti-SMAD4 (Santa Cruz Biotechnology, Santa Cruz, CA, USA; 1:100 and 1:400, respectively), anti-MUC5AC (Merck Millipore, Billerica, MA, USA; 1:100), and anti-CDX2 (BioGenex Laboratories, San Ramon, CA, USA; 1:50). SMAD4 staining was manually performed; all other stainings were run on an automated immunostainer with a iVIEW DAB detection kit (Ventana Medical Systems, Roche, Mannheim, Germany) according to the company's protocols for open procedures with slight modifications.

All tumors were reviewed by three pathologists (AMS, IE and GK) and classified according to the established criteria for the four pancreatic intraductal papillary mucinous neoplasm subtypes. ${ }^{6,31}$ In detail, the gastric subtype was characterized by thick finger-like papillae and cystic lesions lined by columnar cell with mucinous cytoplasm, pyloriclike glands in the periphery of the lesion and expression of MUC5AC; the intestinal subtype was characterized by tall villous papillae, oval and 
hyperchromatic nuclei with pseudostratification and variable amount of apical mucin and expression of MUC2, MUC5AC and CDX2; the pancreato-biliary subtype was characterized by thin branching complex papillae, cuboidal cells with round hyperchromatic nuclei and less mucinous appearance and expression of MUC1 and MUC5AC; and the oncocytic subtype was characterized by thick branching complex papillae with abundant eosinophilic cytoplasm and large round nuclei, as well as focal MUC1 and diffuse MUC5AC expression. In case of discrepant diagnoses, the respective cases were assessed together and the immunohistochemical expression profile served as basis for the final classification.

In intraductal papillary neoplasms of the bile duct, areas with low-grade intraepithelial neoplasia (low-to-moderate grade), high-grade intraepithelial neoplasia or invasive carcinoma were separately evaluated by immunohistochemistry. Invasive carcinomas were subdivided in tubular, colloidmucinous and oncocytic, ${ }^{3,4}$ where applicable. Tumor tissue obtained from biopsy was considered as noninvasive if no clear stromal infiltration was present.

The membranous MUC1 and the cytoplasmic expression of MUC2, MUC5AC and MUC6 were scored according to the percentage of positive cells: negative $(<5 \%)$, focally positive $(5-50 \%)$, and diffusely positive $(>50 \%)$. The nuclear expression of CDX2, TP53 and $\beta$-catenin was scored according to the percentage of positive cells: negative $(<10 \%)$, focally positive $(10-50 \%)$, and diffusely positive $(>50 \%)$. For SMAD4, absence of cytoplasmic and nuclear staining in $>90 \%$ of neoplastic cells was considered as loss of expression. Staining of the above mentioned markers in appropriate control tissues (MUC1, MUC2, MUC5AC, MUC6, CDX2, TP53, $\beta$-catenin) and in normal bile duct epithelium and adjacent stromal cells (SMAD4) served as positive control reactions.

Membranous HER2 expression was assessed using the HercepTest guidelines from DAKO (Dako Denmark A/S, Glostrup, Denmark) for HER2 scoring for gastric cancer in surgical specimens. ${ }^{32}$ All cases with $2+$ and $3+$ scores were further evaluated using fluorescence in situ hybridization, with fluorescent-labeled DNA probes specific for the HER2 locus and the chromosome 17 centromere. The ZytoLight SPEC HER2/CEN17 Dual Color Probe Kit (ZytoVision $\mathrm{GmbH}$, Bremerhaven, Germany) was used according to the manufacturer's protocol with slight modifications. Gene copy number was investigated in hot spot regions in at least 20 cells. HER2 status was expressed as ratio of HER2/CEN 17 score: negative $(<1.8)$, equivocal $(1.8-2.2)$ and amplified $(\geqslant 2.2)$. Membranous EGFR staining was also analyzed according to the HercepTest guidelines for HER2 scoring in gastric carcinoma. Scores between 0-2 were classified as low expression, a score $3+$ as high expression. ${ }^{33}$

\section{Molecular Analysis}

For molecular analysis, DNA extraction was performed separately in manually microdissected lowgrade, high-grade and invasive areas of intraductal papillary neoplasms of the bile duct, where applicable, and in cholangiocarcinomas.

KRAS (exon 2 and 3) and GNAS (exon 8) mutation analysis was performed by a combination of realtime PCR with high-resolution melting analysis as a sensitive screening method using a LightCycler 480 (Roche Diagnostics GmbH, Mannheim and Penzberg, Germany) and direct sequencing of samples, which were identified as positive or suspicious by high resolution melting analysis, using M13r-primer according to the Sanger's method to verify and specify the type of mutation. For mutation analyses of KRAS (exon 2 and 3) and GNAS (exon 8), the following primers were used (all primers in $5^{\prime} \rightarrow 3^{\prime}$ ): KRAS-2-F: CTGAAAATGACTGAATATAAACTTGT GG; KRAS-2-RT: CAGGAAACAGCTATGACCCTGA ATTAGCTGTATCGTCAAGG; KRAS-3-FT: CAGGAA ACAGCTATGACCGACTGTGTTTCTCCCTTCTCAG GATTC; KRAS-3-R: CCAGTCCTCATGTACTGGTCC CTCAT; GNAS-F: CAGGAAACAGCTATGACCCATT GACCTCAATTTTGTTTCAGG; and GNAS-R: TTGG CTTACTGGAAGTTGACTTTG. For Sanger sequencing of GNAS, an independent set of primers was used. ${ }^{16}$

$B R A F$ mutation analysis was performed by mutation-specific real-time PCR, which detects V600E and $\mathrm{V} 600 \mathrm{~K}$ mutations. The following primers were used: BRAF-mut-spec-F: GTAAAAATAGGTGATT TTGGTCTAGCTACAGA and BRAF-R: CTCAATTC TTACCATCCACAAAA.

Loss of heterozygosity of $p 16$ locus was analyzed by real-time PCR followed by advanced relative quantification regarding PCR efficiencies comparing the relative quantity of $p 16$ chromosomal locus (chromosome 19q21) to PAX5 locus (chromosome 9; close to the centromere). DNA from normal colonic muscle tissue served as calibrator for a balanced p16/PAX5 ratio (p16/PAX5 =1). Ratios of p16/PAX5 $<0.7$ were regarded as loss of heterozygosity of the p16 locus. The following primers were used: p16LOH-F: GGACCTGGCTGAGGAGCTG; p16-LOH-R: CATCTATGCGGGCATGGTTAC; PAX5-F: ATCTGTT TCAGGACATGGAGGA, and PAX5-R: CACTATCCT CTGGCGGACTACA.

p16 promoter methylation status was analyzed by the MethyQESD technique ${ }^{34}$ using the following primers: p16-meth-F: AGCACCGGAGGAAGAA AGAG and p16-meth-R: CTGCCTGCTCTCCCCCTCTC.

\section{Statistical Analysis}

Statistical analyses were performed by employing IBM SPSS Statistics software Version 20.0. Survival data were analysed using the Kaplan-Meier method reporting the estimated 1- and 3-year survival probabilities with s.e. Differences in survival curves were assessed by the log-rank test, and Cox's 
proportional hazard models were used in order to determine estimates of hazard ratios with $95 \%$ confidence intervals. Likewise, for relevant estimates of proportions, exact 95\% confidence intervals were reported. All statistical tests were conducted twosided at a 0.05 level of significance. No formal adjustment of $P$-values was considered in order to correct for multiple test issue. However, as the test results of all conducted statistical comparisons were thoroughly reported, an informal adjustment of $P$-values can be performed by reviewing the results. ${ }^{35}$

\section{Results}

\section{Clinicopathological Data}

The data are summarized in Table 1. The mean age (64 years) of patients with intraductal papillary neoplasm of the bile duct at diagnosis did not significantly differ from that of patients with cholangiocarcinoma (67 years). Extrahepatic (including hilar) intraductal papillary neoplasms of the bile duct were more frequent $(60 \%)$ than intrahepatic $(33 \%)$ and combined intraductal papillary neoplasms of the bile duct $(7 \%)$. In detail, most extrahepatic lesions were identified in the intrapancreatic bile duct $(55 \%)$, whereas lesions of the extrapancreatic bile duct and/or cystic duct (33\%) and hilar lesions (11\%) were less common. Cholangiocarcinomas showed a similar distribution $(73 \%$ extrahepatic, 27\% intrahepatic). Two-thirds (69\%) of the intraductal papillary neoplasms of the bile duct were diagnosed at a preinvasive stage. About one-third was associated with an invasive adenocarcinoma, mainly of the tubular type (93\%). Major surgery was performed in 37 of 45 patients $(87 \%)$.

Table 1 Clinicopathological data of patients with intraductal papillary neoplasms of the bile duct or cholangiocarcinomas

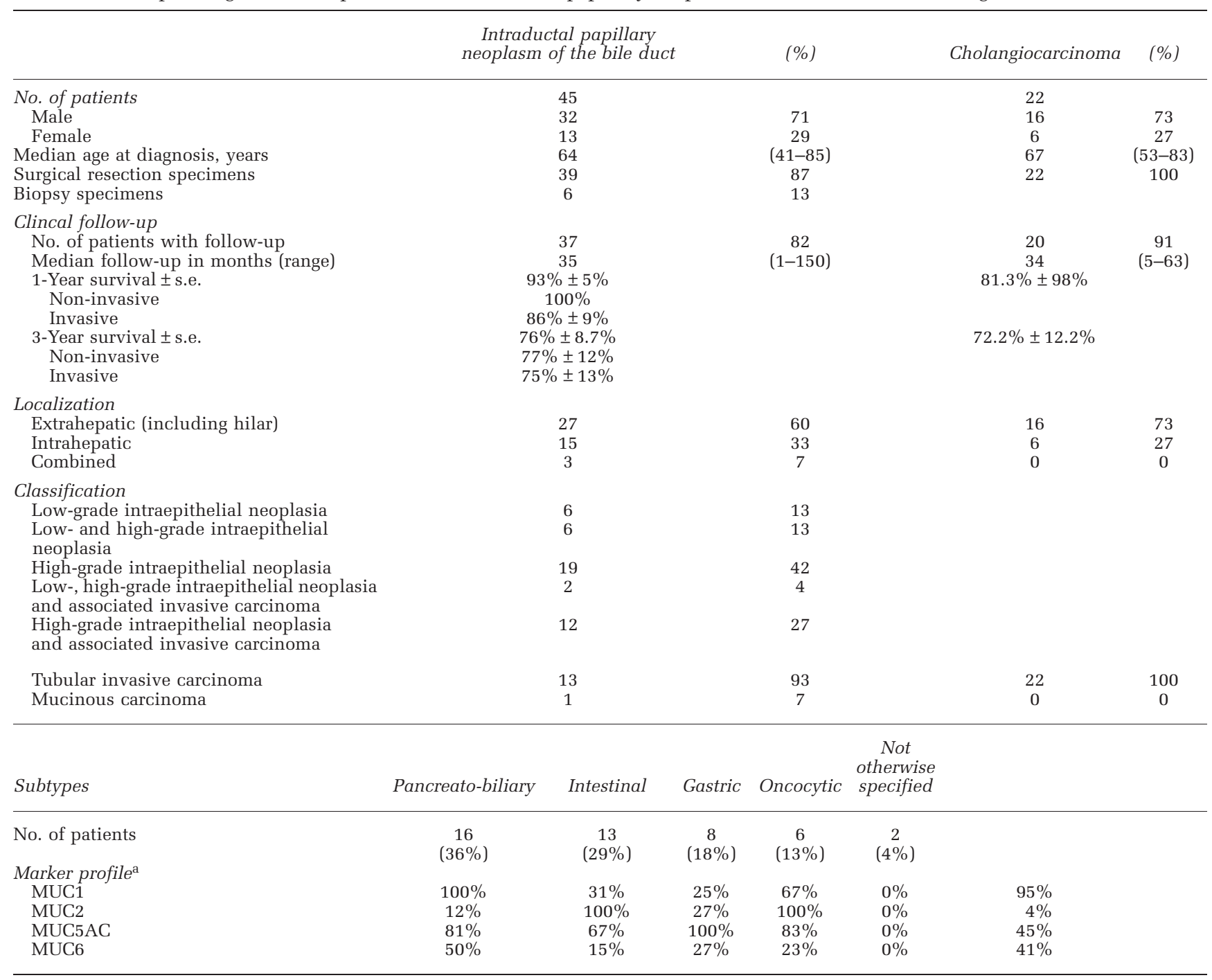

${ }^{\mathrm{a}}$ Focal and diffuse positivity in percentage of all investigated intraductal papillary neoplasms of the bile duct (intraductal lesion only) and cholangiocarcinomas. 
In detail, patients underwent pancreaticoduodenectomy (31\%), hemihepatectomy (28\%), bile duct resection (18\%), segmental liver resection (15\%) and liver transplantation (5\%). No information regarding the surgical treatment was available for one patient.

\section{Pathology}

Grossly, most extrahepatic intraductal papillary neoplasms of the bile duct presented as solid intraluminal tumors $(52 \% ; 0.8-5 \mathrm{~cm})$ or papillary mucosal lesions $(19 \%$; $1-3 \mathrm{~cm})$ dilating the bile duct. Intrahepatic intraductal papillary neoplasms of the bile duct presented more often as cystic lesions $(40 \% ; 0.5-14 \mathrm{~cm})$ than as clearly visible intraluminal $(27 \% ; 2.1-6.5 \mathrm{~cm})$ or intrahepatic tumors $(20 \% ; 4.5-8.5 \mathrm{~cm})$ (Figure 1). Abundant mucin secretion into the duct lumen was observed in a single case (intestinal subtype).

Histologically, subtyping of intraductal papillary neoplasms of the bile duct was possible in 43 (96\%) cases. Pancreato-biliary and intestinal subtypes were most common (36 and 29\%, respectively), followed by gastric and oncocytic subtypes (18 and $13 \%$, respectively) (Table 1, Figure 1). Immunohistochemically, MUC1 was consistently expressed in the pancreato-biliary subtype, MUC2 in the intestinal subtype and MUC5AC in the gastric subtype (Figure 1). Interestingly, CDX2 expression was not a unique feature of intestinal subtype (69\%) but occurred also in a few gastric, oncocytic and pancreato-biliary subtypes $(27,17$ and $6 \%$, respectively). MUC6 was expressed in all the subtypes, with the highest frequency in the pancreato-biliary subtype $(50 \%)$ (Table 1). Two cases $(4 \%)$ showed negativity for all mucin markers but expressed CDX2. As these cases also lacked any of the histological features of one of the four subtypes, they were classified as intraductal papillary neoplasms of the bile duct-not otherwise specified (Table 1).

The pancreato-biliary and oncocytic subtypes showed an equal extra- and intrahepatic distribution (pancreato-biliary: $37 \%$ of all extrahepatic, $40 \%$ of all intrahepatic and absent in combined intraductal papillary neoplasms of the bile duct; oncocytic: $11 \%$
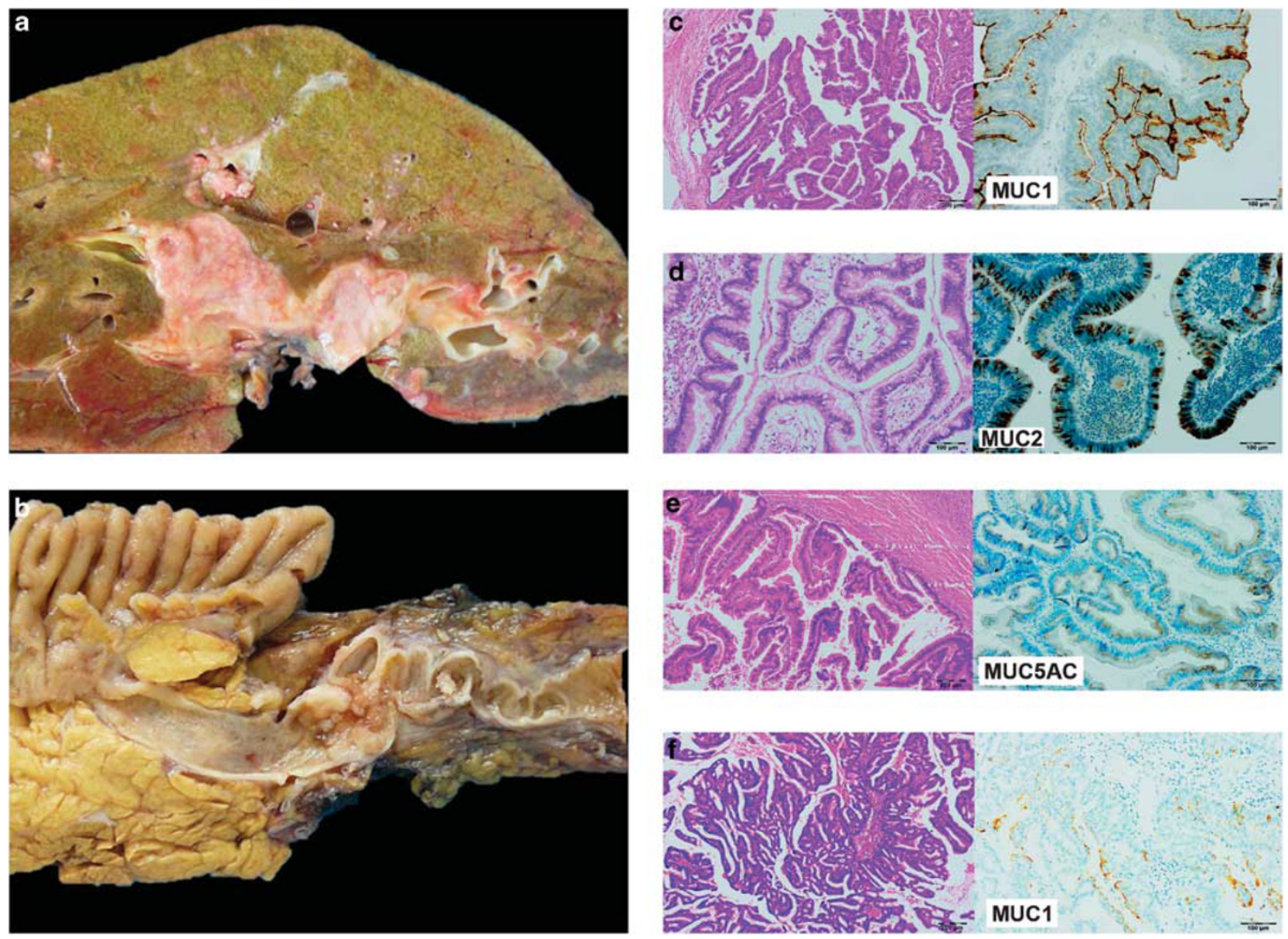

Figure 1 Pathology of intraductal papillary neoplasms of the bile duct. (a) Intrahepatic intraductal papillary neoplasm of the bile duct showing dilated biliary ducts filled with tumor tissue. (b) Extrahepatic intraductal papillary neoplasm of the bile duct with papillary tumor tissue in the ductus choledochus. (c) Pancreato-biliary subtype with diffuse expression of MUC1. (d) Intestinal subtype with diffuse expression of MUC2. (e) Gastric subtype with expression of MUC5AC. (f) Oncocytic subtype with focal expression of MUC1. 
of all extrahepatic, 13\% of all intrahepatic and 33\% of combined intraductal papillary neoplasms of the bile duct). The intestinal subtype was more common outside the liver (intestinal subtype: $30 \%$ of all extrahepatic, $20 \%$ of all intrahepatic and $67 \%$ of combined intraductal papillary neoplasms of the bile duct), while the gastric subtype was more frequent within the liver (gastric subtype: 15\% of all extrahepatic, $27 \%$ of all intrahepatic and absent in combined subtypes). The two intraductal papillary neoplasms of the bile duct-not otherwise specified-were located at extrahepatic sites (not otherwise specified: $7 \%$ of all extrahepatic intraductal papillary neoplasms of the bile duct).

Intraductal papillary neoplasms of the bile duct with low-grade intraepithelial neoplasia were most common in gastric subtype (gastric: $25 \%$, intestinal: 15\%, pancreato-biliary: $13 \%$, oncocytic: $0 \%$, not otherwise specified: $0 \%$ ). Intraductal papillary neoplasms of the bile duct with high-grade intraepithelial neoplasia were commonly detected in oncocytic subtype (oncocytic: 83\%, intestinal: $62 \%$, pancreato-biliary: $50 \%$, gastric: $38 \%$, not otherwise specified: $50 \%$ ). An associated invasive adenocarcinoma was most often found in the gastric (38\%) and pancreato-biliary subtypes (38\%), compared with the intestinal (23\%) and oncocytic (17\%) subtypes. All invasive carcinomas in the pancreatobiliary, gastric and oncocytic subtypes and $2 / 3$ of the intestinal subtype showed tubular features. An invasive mucinous (colloid) adenocarcinoma was only found once and was associated with an intestinal subtype (Table 1).

\section{Follow Up and Survival}

The 1- and 3-year survival rates of intraductal papillary neoplasm of the bile duct patients are summarized in Table 1. Comparison of the overall survival rates revealed slightly worse prognosis for

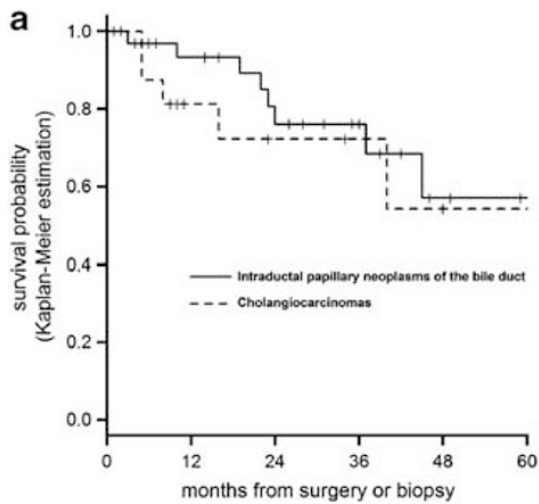

cholangiocarcinoma patients compared with intraductal papillary neoplasm of the bile duct patients (hazard ratio: 1.40; 95\% confidence interval: 0.46-4.30), a difference which, however, was not statistically significant ( $P$-value log-rank test: 0.552; Figure 2). Subgroup comparison of patients with intraductal papillary neoplasm of the bile duct with invasion versus those without invasion showed twofold higher risk of death in the first group. However, as reflected by the wide confidence interval, the risk estimation had a high uncertainty (hazard ratio: 2; 95\% confidence interval: 0.48-8.02; $P=0.327$; Figure 2). Likewise, no statistically significant prognostic differences could be detected between different histological subtypes ( $P$-value logrank test: 0.84, data not shown). During the followup of patients with histologically non-invasive intraductal papillary neoplasm of the bile duct, two patients developed invasive carcinoma (probably due to incomplete resection or multifocality) and died of the disease. In detail, one patient who clinically presented invasion of the vena cava had been treated with hemihepatectomy. However in the resection specimen, there was no evidence of invasive growth. During the follow-up, the patient developed invasive carcinoma and died of disease (10 months after initial diagnosis). As the patient clinically presented with advance invasive carcinoma, the patient was classified as patient with invasive intraductal papillary neoplasm for the survival analysis. One patient died 22 months after the initial diagnosis, which was made on biopsy and followed by palliative treatment without major surgery.

\section{Genetic Findings}

A summary of the genetic findings is presented in Tables 2 and 3 . A detailed list of individual molecular changes is given in Table 4 .

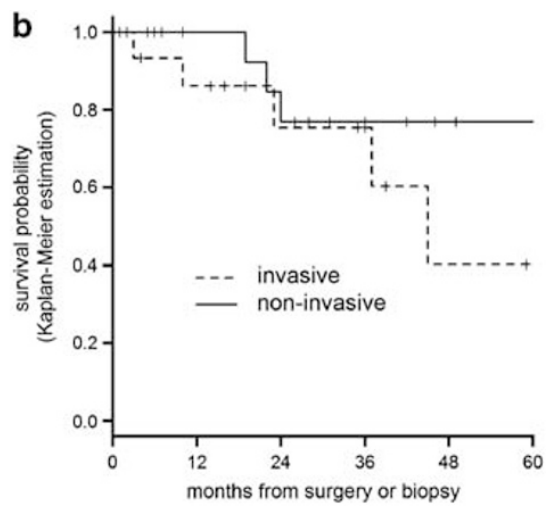

Figure 2 Kaplan-Meier survival curves. (a) Comparison of postoperative overall survival revealed slightly worse, however, not statistically significant, prognosis for patients with cholangiocarcinoma compared with patients with intraductal papillary neoplasm of the bile duct (hazard ratio: $1.4 ; 95 \%$ confidence interval: $0.46-4.30 ; P=0.552$ ). (b) Likewise, no significant differences in survival prognosis were detected in subgroup comparison of intraductal papillary neoplasm of the bile duct patients with invasive carcinoma versus intraductal papillary neoplasm of the bile duct patients without invasion (hazard ratio: 2; 95\% confidence interval: $0.48-8.02$; $P=0.327$ ). However, subgroup comparison of patients with intraductal papillary neoplasm of the bile duct with invasion versus those without invasion showed twofold higher risk of death in the first group. 
Table 2 Molecular events in progression from low-grade to high-grade intraductal papillary neoplasm of the bile duct and invasive cancer compared with conventional cholangiocarcinoma

\begin{tabular}{|c|c|c|c|c|}
\hline \multirow[b]{2}{*}{$\begin{array}{l}\text { Genes/proteins } \\
\text { with changes }\end{array}$} & \multicolumn{3}{|c|}{ Intraductal papillary neoplasm of the bile duct } & \multirow{2}{*}{ 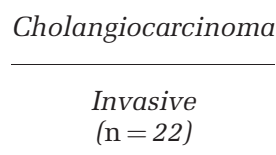 } \\
\hline & $\begin{array}{l}\text { Low-grade intraepithelial } \\
\text { neoplasia }(\mathrm{n}=14)\end{array}$ & $\begin{array}{l}\text { High-grade intraepithelial } \\
\text { neoplasia }(\mathrm{n}=39)\end{array}$ & $\begin{array}{l}\text { Invasive carcinoma } \\
(\mathrm{n}=14)\end{array}$ & \\
\hline TP53 & $36 \%(5 / 14)$ & $68 \%(26 / 38)$ & $85 \%(11 / 13)$ & $64 \%(14 / 22)$ \\
\hline KRAS (exon 2) & $50 \%(7 / 14)$ & $26 \%(10 / 38)$ & $8 \%(1 / 13)$ & $14 \%(3 / 22)$ \\
\hline KRAS (exon 3) & $8 \%(1 / 13)$ & $0 \%(0 / 36)$ & $0 \%(0 / 13)$ & $0 \%(0 / 22)$ \\
\hline$B R A F$ & $0 \%(0 / 12)$ & $0 \%(0 / 35)$ & $0 \%(0 / 13)$ & $0 \%(0 / 22)$ \\
\hline p16 (loss of heterozygosity) & $8 \%(1 / 12)$ & $15 \%(5 / 33)$ & $8 \%(1 / 12)$ & $27 \%(6 / 22)$ \\
\hline p16 (promoter methylation) & $10 \%(1 / 10)$ & $13 \%(3 / 24)$ & $9 \%(1 / 11)$ & $30 \%(3 / 10)$ \\
\hline$\beta$-Catenin & $14 \%(2 / 14)$ & $8 \%(3 / 38)$ & $0 \%(0 / 13)$ & $0 \%(0 / 22)$ \\
\hline GNAS & $8 \%(1 / 13)$ & $0 \%(0 / 36)$ & $0 \%(0 / 13)$ & $0 \%(0 / 22)$ \\
\hline SMAD4 & $0 \%(0 / 14)$ & $8 \%(3 / 38)$ & $15 \%(2 / 13)$ & $14 \%(3 / 22)$ \\
\hline HER2 & $0 \%(0 / 14)$ & $5 \%(2 / 38)$ & $0 \%(0 / 13)$ & $0 \%(0 / 22)$ \\
\hline EGFR & $0 \%(0 / 14)$ & $3 \%(1 / 38)$ & $8 \%(1 / 13)$ & $5 \%(1 / 22)$ \\
\hline
\end{tabular}

Table 3 Genetic changes in intraductal papillary neoplasms of the bile duct, cholangiocarcinomas and subtypes of intraductal papillary neoplasm of the bile duct

\begin{tabular}{|c|c|c|c|c|c|c|c|c|c|c|}
\hline & No. of cases & TP53 & KRAS & $B R A F$ & p16 & $\beta$-Catenin & SMAD4 & GNAS & HER2 & EGFR \\
\hline \multicolumn{11}{|c|}{ Total intraductal papillary neoplasms of the bile duct } \\
\hline & 45 & $67 \%(30 / 45)$ & $36 \%(16 / 45)$ & $0 \%(0 / 43)$ & $24 \%(10 / 42)$ & $9 \%(4 / 45)$ & $7 \%(3 / 45)$ & $2 \%(1 / 44)$ & $4 \%(2 / 45)$ & $2 \%(1 / 45)$ \\
\hline \multicolumn{11}{|l|}{ Total cholangiocarcinomas } \\
\hline & 22 & $64 \%(14 / 22)$ & $14 \%(3 / 22)$ & $0 \%(0 / 22)$ & $36 \%(8 / 22)$ & $0 \%(0 / 22)$ & $14 \%(3 / 22)$ & $0 \%(0 / 22)$ & $0 \%(0 / 22)$ & $5 \%(1 / 22)$ \\
\hline \multicolumn{11}{|c|}{ Intraductal papillary neoplasms of the bile duct } \\
\hline Extrahepatic & 27 & $81 \%(22 / 27)$ & $30 \%(8 / 27)$ & $0 \%(0 / 26)$ & $33 \%(9 / 27)$ & $11 \%(3 / 27)$ & $7 \%(2 / 27)$ & $0 \%(0 / 26)$ & $7 \%(2 / 27)$ & $4 \%(1 / 27)$ \\
\hline Intrahepatic & 15 & $40 \%(6 / 15)$ & $47 \%(7 / 15)$ & $0 \%(0 / 14)$ & $0 \%(0 / 12)$ & $7 \%(1 / 15)$ & $7 \%(1 / 15)$ & $0 \%(0 / 15)$ & $0 \%(0 / 15)$ & $0 \%(0 / 15)$ \\
\hline Combined & 3 & $67 \%(2 / 3)$ & $33 \%(1 / 3)$ & $0 \%(0 / 3)$ & $33 \%(1 / 3)$ & $0 \%(0 / 3)$ & $0 \%(0 / 3)$ & $33 \%(1 / 3)$ & $0 \%(0 / 3)$ & $0 \%(0 / 3)$ \\
\hline \multicolumn{11}{|c|}{ Intraductal papillary neoplasms of the bile duct subtypes } \\
\hline Pancreato-biliary & 16 & $75 \%(12 / 16)$ & $25 \%(4 / 16)$ & $0 \%(0 / 16)$ & $20 \%(3 / 15)$ & $6 \%(1 / 16)$ & $13 \%(2 / 16)$ & $0 \%(0 / 16)$ & $13 \%(2 / 16)$ & $0 \%(0 / 16)$ \\
\hline Intestinal & 13 & $69 \%(9 / 13)$ & $46 \%(6 / 13)$ & $0 \%(0 / 11)$ & $27 \%(3 / 11)$ & $8 \%(1 / 13)$ & $0 \%(0 / 13)$ & $8 \%(1 / 12)$ & $0 \%(0 / 13)$ & $0 \%(0 / 13)$ \\
\hline Gastric & 8 & $25 \%(2 / 8)$ & $62 \%(5 / 8)$ & $0 \%(0 / 8)$ & $25 \%(2 / 8)$ & $13 \%(1 / 8)$ & $13 \%(1 / 8)$ & $0 \%(0 / 8)$ & $0 \%(0 / 8)$ & $13 \%(1 / 8)$ \\
\hline Oncocytic & 6 & $83 \%(5 / 6)$ & $0 \%(0 / 6)$ & $0 \%(0 / 6)$ & $33 \%(2 / 6)$ & $0 \%(0 / 6)$ & $0 \%(0 / 6)$ & $0 \%(0 / 6)$ & $0 \%(0 / 6)$ & $0 \%(0 / 6)$ \\
\hline Not otherwise specified & 2 & $100 \%(2 / 2)$ & $50 \%(1 / 2)$ & $0 \%(0 / 2)$ & $0 \%(0 / 2)$ & $50 \%(1 / 2)$ & $0 \%(0 / 2)$ & $0 \%(0 / 2)$ & $0 \%(0 / 2)$ & $0 \%(0 / 2)$ \\
\hline
\end{tabular}

The TP53 gene was tested regarding its mutational inactivation using the immunohistochemical analysis of the nuclear TP53 accumulation. In intraductal papillary neoplasms of the bile duct, the frequency of nuclear TP53 expression progressively increased from low-grade intraepithelial neoplasia to invasive carcinoma. Intraductal papillary neoplasms of the bile duct and cholangiocarcinomas showed comparable high frequencies of TP53 expression (Tables 2 and 3).

Activating KRAS mutations were detected in the hotspot regions of exons 2 and 3 in $36 \%$ of all intraductal papillary neoplasms of the bile duct compared with $14 \%$ in cholangiocarcinomas. They were much more common in low-grade $(57 \%)$ than high-grade lesions and associated invasive carcinomas (26 and $8 \%$, respectively). BRAF mutations were not detected (Tables 2 and 3 ).

The $p 16$ gene product acts as regulator of the cell cycle and is inactivated by loss of heterozygos- ity of locus 9p21, promoter methylation or both. Inactivation of $p 16$ was exclusively detected in extrahepatic and combined intraductal papillary neoplasms of the bile duct and was more frequent in cholangiocarcinomas $24 \%$ of total intraductal papillary neoplasms of the bile duct versus $36 \%$ of cholangiocarcinoma; Tables 2 and 3 ). In intraductal papillary neoplasms of the bile duct, $p 16$ changes were detected in low-grade, high-grade and invasive lesions (Table 2). Synchronous p16 loss of heterozygosity and promoter hypermethylation was present in one cholangiocarcinoma.

$\beta$-Catenin protein accumulation in the nucleus, indicating $\beta$-Catenin mutation or genetic alteration of the Wnt/ $\beta$-catenin pathway, occurred in intraductal papillary neoplasms of the bile duct only in non-invasive lesions but was absent in associated carcinomas and cholangiocarcinomas (Tables 2 and 3). 
Table 4 Detailed list of individual molecular changes

Intraductal papillary neoplasms of the bile duct

\begin{tabular}{|c|c|c|c|c|c|c|c|c|c|c|c|c|c|c|}
\hline & Subtype & $\begin{array}{l}\text { Intraepithelial } \\
\text { neoplasia }\end{array}$ & TP53 & $\begin{array}{c}\text { KRAS } \\
\text { (exon 2) }\end{array}$ & $\begin{array}{c}\text { KRAS } \\
\text { (exon 3) }\end{array}$ & $\begin{array}{c}\text { GNAS } \\
\text { (exon 8) }\end{array}$ & $B R A F$ & $\begin{array}{l}\text { p16 } \\
\text { promoter }\end{array}$ & $\begin{array}{l}\text { p16 loss of } \\
\text { heterozygosity }\end{array}$ & SMAD4 & $\begin{array}{l}\text { HER2 immunohisto- } \\
\text { chemistry }\end{array}$ & $\begin{array}{l}\text { HER2 fluorescence } \\
\text { in situ hybridization }\end{array}$ & $E G F R$ & $\beta$-Catenin \\
\hline 1 & Intestinal & High-grade & Dif & p.G12D & wt & wt & wt & Unmeth & LOH & No loss & $2+$ & No ampl & Low & Neg \\
\hline \multirow[t]{2}{*}{2} & Intestinal & Low-grade & Neg & p.G12A & wt & wt & wt & Unmeth & wt & No loss & $2+$ & No ampl & Low & Neg \\
\hline & & High-grade & Dif & p.G12A & wt & wt & wt & Unmeth & wt & No loss & $2+$ & No ampl & Low & Neg \\
\hline 3 & Intestinal & Low-grade & Foc & wt & wt & wt & wt & Unmeth & wt & No loss & $2+$ & No ampl & Low & Neg \\
\hline 4 & Intestinal & High-grade & Dif & wt & NA & NA & NA & NA & NA & No loss & $2+$ & No ampl & Low & $\mathrm{Neg}$ \\
\hline 5 & Gastric & Low-grade & Dif & wt & p.Q61H & wt & wt & Unmeth & LOH & No loss & $\mathrm{Neg}$ & No ampl & Low & $\mathrm{Neg}$ \\
\hline 6 & Pancreato-biliary & High-grade & Dif & wt & wt & wt & wt & Unmeth & wt & No loss & $2+$ & No ampl & Low & Neg \\
\hline \multirow[t]{3}{*}{7} & Pancreato-biliary & Low-grade & Dif & wt & wt & wt & wt & Meth & wt & No loss & $2+$ & No ampl & Low & Neg \\
\hline & & High-grade & Dif & wt & wt & wt & wt & Meth & wt & No loss & $2+$ & No ampl & Low & Neg \\
\hline & & Invasive carcinoma & Dif & wt & $\mathrm{wt}$ & wt & wt & Unmeth & wt & No loss & $\mathrm{Neg}$ & & Low & $\mathrm{Neg}$ \\
\hline \multirow[t]{2}{*}{8} & Gastric & High-grade & Neg & wt & $\mathrm{wt}$ & wt & wt & Unmeth & LOH & No loss & $2+$ & No ampl & High & Neg \\
\hline & & Invasive carcinoma & Neg & wt & wt & wt & wt & Unmeth & wt & No loss & $2+$ & No ampl & High & $\mathrm{Neg}$ \\
\hline \multirow[t]{2}{*}{9} & Intestinal & High-grade & Neg & p.G13D & wt & wt & wt & Unmeth & wt & No loss & $2+$ & No ampl & Low & Foc \\
\hline & & Invasive carcinoma & Dif & wt & $\mathrm{wt}$ & wt & wt & Unmeth & wt & No loss & $2+$ & No ampl & Low & Neg \\
\hline \multirow[t]{2}{*}{10} & Gastric & Low-grade & Neg & p.G12S & wt & wt & wt & Unmeth & wt & No loss & $\mathrm{Neg}$ & & Low & Foc \\
\hline & & High-grade & Neg & p.G12S & NA & NA & NA & NA & NA & No loss & $\mathrm{Neg}$ & & Low & Foc \\
\hline 11 & Intestinal & High-grade & Dif & wt & wt & wt & wt & Unmeth & $\mathrm{LOH}$ & No loss & $2+$ & NA & Low & $\mathrm{Neg}$ \\
\hline \multirow[t]{2}{*}{12} & Oncocytic & High-grade & Dif & wt & wt & wt & wt & Unmeth & wt & No loss & $\mathrm{Neg}$ & & Low & Neg \\
\hline & & Invasive carcinoma & Dif & wt & wt & wt & wt & Unmeth & wt & No loss & Neg & & Low & Neg \\
\hline 13 & Oncocytic & High-grade & Dif & wt & wt & wt & wt & Unmeth & wt & No loss & Neg & & Low & Neg \\
\hline \multirow[t]{2}{*}{14} & Pancreato-biliary & High-grade & Dif & wt & wt & wt & wt & NA & NA & No loss & $3+$ & Ampl & Low & Neg \\
\hline & & Invasive carcinoma & NA & NA & NA & NA & NA & NA & NA & NA & NA & $\mathrm{NA}$ & NA & $\mathrm{NA}$ \\
\hline \multirow[t]{2}{*}{15} & Intestinal & Low-grade & Neg & p.G12D & NA & NA & NA & NA & NA & No loss & Neg & & $\mathrm{NA}$ & Neg \\
\hline & & High-grade & NA & p.G12D & wt & wt & wt & NA & wt & NA & NA & & Low & NA \\
\hline 16 & Pancreato-biliary & Low-grade & Foc & p.G12D & wt & wt & wt & Unmeth & wt & No loss & $2+$ & No ampl & Low & Foc \\
\hline 17 & Pancreato-biliary & High-grade & Neg & wt & wt & wt & wt & NA & wt & Loss & Neg & & Low & Neg \\
\hline \multirow[t]{2}{*}{18} & Pancreato-biliary & Low-grade & Neg & $\mathrm{wt}$ & $\mathrm{wt}$ & wt & wt & Unmeth & wt & No loss & $2+$ & No ampl & Low & Neg \\
\hline & & High-grade & Foc & $\mathrm{wt}$ & $\mathrm{wt}$ & wt & wt & NA & wt & No loss & $\mathrm{Neg}$ & & Low & $\mathrm{Neg}$ \\
\hline \multirow{2}{*}{19} & Pancreato-biliary & High-grade & Foc & p.G12V & $\mathrm{wt}$ & wt & wt & Unmeth & wt & No loss & Neg & & Low & $\mathrm{Neg}$ \\
\hline & & Invasive carcinoma & Dif & wt & wt & wt & wt & Unmeth & wt & No loss & $2+$ & No ampl & Low & Neg \\
\hline 20 & Pancreato-biliary & Highgrade & Dif & wt & wt & wt & wt & Unmeth & wt & No loss & $2+$ & No ampl & Low & Neg \\
\hline 21 & Oncocytic & High-grade & Foc & wt & wt & wt & wt & Meth & wt & No loss & $\mathrm{Neg}$ & & Low & Neg \\
\hline 22 & Pancreato-biliary & High-grade & Neg & wt & wt & wt & wt & Meth & wt & No loss & $\mathrm{Neg}$ & & Low & Neg \\
\hline \multirow[t]{3}{*}{23} & Gastric & Low-grade & Neg & wt & wt & wt & wt & Unmeth & wt & No loss & $\mathrm{Neg}$ & & Low & Neg \\
\hline & & High-grade & Neg & wt & wt & wt & wt & Unmeth & wt & No loss & $2+$ & No ampl & Low & Neg \\
\hline & & Invasive carcinoma & Neg & p.G12D & $\mathrm{wt}$ & wt & wt & Unmeth & wt & No loss & $2+$ & No ampl & Low & $\mathrm{Neg}$ \\
\hline \multirow[t]{2}{*}{24} & Gastric & High-grade & Dif & wt & $\mathrm{wt}$ & wt & wt & Unmeth & wt & Loss & $2+$ & No ampl & Low & Neg \\
\hline & & Invasive carcinoma & Dif & wt & $\mathrm{wt}$ & wt & wt & Unmeth & wt & Loss & $2+$ & No ampl & Low & $\mathrm{Neg}$ \\
\hline 25 & Intestinal & Low-grade & Neg & wt & wt & p.R201H & wt & Unmeth & wt & No loss & Neg & & Low & Neg \\
\hline 26 & Gastric & High-grade & Neg & wt & $\mathrm{wt}$ & wt & wt & NA & wt & No loss & Neg & & Low & Neg \\
\hline 27 & Oncocytic & High-grade & Foc & wt & wt & wt & wt & Unmeth & $\mathrm{LOH}$ & No loss & $\mathrm{Neg}$ & & Low & Neg \\
\hline \multirow[t]{2}{*}{28} & Pancreato-biliary & High-grade & Foc & wt & wt & wt & wt & Unmeth & wt & No loss & Neg & & Low & Neg \\
\hline & & Invasive carcinoma & Foc & wt & wt & wt & wt & Unmeth & wt & No loss & Neg & & Low & Neg \\
\hline \multirow[t]{2}{*}{29} & Pancreato-biliary & High-grade & Dif & wt & wt & wt & wt & Unmeth & wt & No loss & $3+$ & Ampl & Low & $\mathrm{Neg}$ \\
\hline & & Invasive carcinoma & Dif & wt & wt & wt & wt & Meth & wt & No loss & $\mathrm{Neg}$ & & Low & Neg \\
\hline \multirow[t]{2}{*}{30} & Not otherwise specified & High-grade & Dif & wt & wt & wt & wt & Unmeth & LOH & No loss & $2+$ & No ampl & Low & Neg \\
\hline & & $\begin{array}{l}\text { Invasive } \\
\text { carcinoma }\end{array}$ & Dif & wt & $\mathrm{wt}$ & wt & wt & Unmeth & LOH & No loss & $2+$ & No ampl & Low & $\mathrm{Neg}$ \\
\hline 31 & Not otherwise specified & Low-grade & Neg & p.G12V & wt & wt & wt & NA & wt & No loss & Neg & & Low & Neg \\
\hline & & High-grade & Dif & NA & NA & NA & NA & NA & NA & No loss & Neg & & Low & $\mathrm{Neg}$ \\
\hline 32 & Intestinal & High-grade & Foc & wt & wt & wt & wt & Unmeth & wt & No loss & $2+$ & No ampl & Low & Neg \\
\hline & & Invasive carcinoma & Foc & wt & wt & wt & wt & Unmeth & wt & No loss & Neg & & Low & Neg \\
\hline 33 & Pancreato-biliary & High-grade & Neg & p.G12V & wt & wt & wt & $\mathrm{NA}$ & wt & No loss & Neg & & Low & Neg \\
\hline
\end{tabular}




\begin{tabular}{|c|c|c|c|c|c|c|c|c|c|c|c|c|c|c|}
\hline \multicolumn{15}{|c|}{ Intraductal papillary neoplasms of the bile duct } \\
\hline & Subtype & $\begin{array}{l}\text { Intraepithelial } \\
\text { neoplasia }\end{array}$ & TP53 & $\begin{array}{l}\text { KRAS } \\
(\text { exon 2) }\end{array}$ & $\begin{array}{l}\text { KRAS } \\
\text { (exon 3) }\end{array}$ & $\begin{array}{l}\text { GNAS } \\
\text { (exon 8) }\end{array}$ & $B R A F$ & $\begin{array}{l}\text { p16 } \\
\text { promoter }\end{array}$ & $\begin{array}{l}\text { p16 loss of } \\
\text { heterozygosity }\end{array}$ & SMAD4 & $\begin{array}{l}\text { HER2 immunohisto- } \\
\text { chemistry }\end{array}$ & $\begin{array}{l}\text { HER2 fluorescence } \\
\text { in situ hybridization }\end{array}$ & EGFR & $\beta$-Catenin \\
\hline 34 & Oncocytic & High-grade & Foc & wt & wt & wt & wt & NA & wt & No loss & $\mathrm{Neg}$ & & Low & $\mathrm{Neg}$ \\
\hline 35 & Intestinal & High-grade & $\mathrm{Neg}$ & wt & wt & wt & wt & NA & wt & No loss & $\mathrm{Neg}$ & & Low & $\mathrm{Neg}$ \\
\hline 36 & Pancreato-biliary & High-grade & Foc & wt & wt & wt & wt & NA & wt & No loss & $2+$ & NA & Low & Neg \\
\hline 37 & Intestinal & High-grade & $\mathrm{Neg}$ & p.G12A & wt & wt & wt & Unmeth & $\mathrm{wt}$ & No loss & $\mathrm{Neg}$ & & Low & $\mathrm{Neg}$ \\
\hline 38 & Pancreato-biliary & Low-grade & $\mathrm{Neg}$ & wt & wt & wt & wt & Unmeth & wt & No loss & Neg & & Low & Neg \\
\hline \multirow[t]{2}{*}{39} & Intestinal & Low-grade & Dif & p.G12A & wt & wt & $\mathrm{NA}$ & NA & wt & No loss & $2+$ & No ampl & Low & $\mathrm{Neg}$ \\
\hline & & High-grade & Dif & wt & wt & wt & NA & NA & wt & No loss & Neg & & Low & Neg \\
\hline \multirow[t]{2}{*}{40} & Intestinal & High-grade & Dif & wt & wt & wt & wt & NA & NA & No loss & Neg & & Low & Neg \\
\hline & & Invasive carcinoma & Dif & wt & wt & wt & wt & NA & $\mathrm{NA}$ & No loss & $\mathrm{Neg}$ & & Low & $\mathrm{Neg}$ \\
\hline 41 & Gastric & High-grade & $\mathrm{Neg}$ & p.G12S & wt & wt & wt & NA & wt & No loss & $\mathrm{Neg}$ & & Low & $\mathrm{Neg}$ \\
\hline 42 & Gastric & Low-grade & $\mathrm{Neg}$ & p.G12C & wt & wt & wt & NA & $\mathrm{wt}$ & No loss & Neg & & Low & Neg \\
\hline \multirow[t]{2}{*}{43} & Pancreato-biliary & High-grade & Foc & wt & wt & wt & wt & Unmeth & wt & Loss & $2+$ & NA & Low & $\mathrm{Neg}$ \\
\hline & & Invasive carcinoma & Dif & wt & wt & wt & wt & NA & wt & Loss & $2+$ & NA & Low & $\mathrm{Neg}$ \\
\hline 44 & Oncocytic & High-grade & $\mathrm{Neg}$ & wt & wt & wt & wt & Unmeth & wt & No loss & $2+$ & NA & Low & $\mathrm{Neg}$ \\
\hline \multirow[t]{3}{*}{45} & Pancreato-biliary & High-grade & Dif & p.G12V & wt & wt & wt & Unmeth & wt & No loss & $\mathrm{Neg}$ & & Low & Neg \\
\hline & & & \multicolumn{12}{|c|}{ Cholangiocarcinomas } \\
\hline & & & TP53 & $\begin{array}{c}\text { KRAS } \\
(\operatorname{exon} 2)\end{array}$ & $\begin{array}{c}\text { KRAS } \\
\text { (exon 3) }\end{array}$ & $\begin{array}{c}\text { GNAS } \\
\text { (exon 8) }\end{array}$ & $\begin{array}{l}\text { BRAF } \\
\text { (exon 15) }\end{array}$ & $\begin{array}{l}\text { p16 } \\
\text { promoter }\end{array}$ & $\begin{array}{l}\text { p16 loss of } \\
\text { heterozygosity }\end{array}$ & SMAD4 & $\begin{array}{l}\text { HER2 immunohisto- } \\
\text { chemistry }\end{array}$ & $\begin{array}{l}\text { HER2 fluorescence } \\
\text { in situ hybridization }\end{array}$ & $E G F R$ & $\beta$-Catenin \\
\hline 1 & & & Dif & wt & wt & wt & wt & NA & wt & Loss & Neg & & Low & Neg \\
\hline 2 & & & Dif & wt & wt & wt & wt & Meth & wt & No loss & $2+$ & No ampl & Low & $\mathrm{Neg}$ \\
\hline 3 & & & Neg & wt & wt & wt & wt & Meth & $\mathrm{LOH}$ & No loss & $\mathrm{Neg}$ & & Low & $\mathrm{Neg}$ \\
\hline 4 & & & Neg & wt & wt & wt & $\mathrm{wt}$ & Unmeth & wt & No loss & $2+$ & No ampl & Low & Neg \\
\hline 5 & & & Dif & wt & wt & wt & wt & Unmeth & wt & No loss & $2+$ & No ampl & Low & Neg \\
\hline 6 & & & Dif & wt & wt & wt & wt & Unmeth & wt & No loss & $2+$ & No ampl & Low & $\mathrm{Neg}$ \\
\hline 7 & & & Neg & wt & wt & wt & $\mathrm{wt}$ & $\mathrm{NA}$ & wt & Loss & $2+$ & No ampl & Low & $\mathrm{Neg}$ \\
\hline 8 & & & Neg & wt & wt & wt & $\mathrm{wt}$ & Unmeth & wt & No loss & $\mathrm{Neg}$ & & Low & $\mathrm{Neg}$ \\
\hline 10 & & & Foc & wt & wt & wt & wt & Unmeth & $\mathrm{LOH}$ & No loss & $\mathrm{Neg}$ & & Low & $\mathrm{Neg}$ \\
\hline 11 & & & Neg & p.G12V & wt & wt & $\mathrm{wt}$ & NA & LOH & No loss & Neg & & Low & $\mathrm{Neg}$ \\
\hline 12 & & & Neg & p.G12D & wt & wt & $\mathrm{wt}$ & NA & LOH & No loss & $2+$ & No ampl & High & $\mathrm{Neg}$ \\
\hline 13 & & & Foc & wt & wt & wt & wt & NA & wt & No loss & $\mathrm{Neg}$ & & Low & $\mathrm{Neg}$ \\
\hline 14 & & & Neg & wt & wt & wt & wt & NA & wt & No loss & $\mathrm{Neg}$ & & Low & $\mathrm{Neg}$ \\
\hline 15 & & & Dif & wt & wt & wt & wt & Unmeth & LOH & No loss & $2+$ & No ampl & Low & Neg \\
\hline 16 & & & Dif & wt & wt & wt & wt & NA & wt & No loss & $2+$ & No ampl & Low & $\mathrm{Neg}$ \\
\hline 17 & & & Dif & wt & wt & wt & wt & Meth & wt & Loss & Neg & & Low & $\mathrm{Neg}$ \\
\hline 18 & & & Dif & wt & wt & wt & wt & NA & wt & No loss & $2+$ & No ampl & Low & $\mathrm{Neg}$ \\
\hline 19 & & & Dif & wt & wt & wt & $\mathrm{wt}$ & NA & wt & No loss & $2+$ & No ampl & Low & $\mathrm{Neg}$ \\
\hline 20 & & & Foc & p.G12D & wt & wt & wt & NA & wt & No loss & $\mathrm{Neg}$ & & Low & $\mathrm{Neg}$ \\
\hline 21 & & & Foc & wt & wt & wt & wt & Unmeth & wt & No loss & $\mathrm{Neg}$ & & Low & $\mathrm{Neg}$ \\
\hline 22 & & & Dif & $\mathrm{wt}$ & wt & wt & $\mathrm{wt}$ & NA & LOH & No loss & $2+$ & No ampl & Low & $\mathrm{Neg}$ \\
\hline 23 & & & Neg & wt & wt & wt & wt & $\mathrm{NA}$ & wt & No loss & $\mathrm{Neg}$ & & Low & Neg \\
\hline
\end{tabular}


GNAS mutations in the hot-spot regions of exon 8 were rare. An activating mutation in codon 201 (p.R201H) was only found in a single intraductal papillary neoplasm of the bile duct of the intestinal subtype with low-grade intraepithelial neoplasia (Tables 2 and 3, Figure 3).

SMAD4/DPC4 protein accumulation in the nucleus indicates a wild-type status. Immunohistochemical loss of SMAD4, correlating with an inactivating mutation, ${ }^{36,37}$ only occurred in highgrade and invasive intraductal papillary neoplasms of the bile duct, showing here increasing frequency (Table 2, Figure 3). Loss of SMAD4 was less common in intraductal papillary neoplasms of the bile duct than cholangiocarcinomas (7 versus 14\%; Tables 2 and 3).

HER2 overexpression, corresponding to score $3+$ and corresponding to fluorescence in situ hybridization high-level gene amplification, was only observed in two intraductal papillary neoplasms of the bile duct with high-grade intraepithelial neoplasia. In one of two intraductal papillary neoplasms of the bile duct, HER2 was only expressed in the dysplastic area but not in the invasive component. Fluorescence in situ hybridization analysis of cases that scored $2+$ was possible in 17 of 21 tumors ( $81 \%$ ) and revealed no HER2 gene amplification (for details, see Table 4).

EGFR overexpression was observed in one intraductal papillary neoplasm of the bile duct with high-grade intraepithelial neoplasia and its associated invasive component. Intraductal papillary neoplasms of the bile duct were less affected than cholangiocarcinomas ( 2 versus $5 \%$; Tables 2 and 3 ).

Table 3 summarizes the data on the mutational status in intraductal papillary neoplasms of the bile duct and their subtypes and in cholangiocarcinomas. Of particular interest is the absence of GNAS mutations from all but one intraductal papillary neoplasm of the bile duct (that showed an intestinal subtype) and the absence of KRAS mutations from intraductal papillary neoplasms of the bile duct of the oncocytic subtype.

\section{Discussion}

Intraductal papillary neoplasms of the bile duct are rare precursors of invasive intra- and extrahepatic bile duct carcinomas and are considered the biliary counterpart of pancreatic intraductal papillary mucinous neoplasms. ${ }^{2,5}$ Due to the small number of cases and due to the absence of a uniform classification until recently, intraductal papillary neoplasms of the bile duct are still poorly characterized regarding their mutational status in relation to their development from low-grade intraductal neoplasms to invasive adenocarcinomas. It is also not well known whether they differ biologically from cholangiocarcinomas or from each other if they are stratified by subtype.
The data of our study suggest that the oncogenetic profile of intraductal papillary neoplasms of the bile duct follows the stepwise progression from lowgrade intraductal papillary dysplasia at its beginning to invasive adenocarcinoma at its end. TP53, KRAS and p16 (in decreasing order of frequency) were the most commonly affected genes/proteins in the intraductal papillary neoplasms of the bile duct with low-grade intraepithelial neoplasia, and inactivation of $p 16$ was interestingly found only in extrahepatic or combined intraductal papillary neoplasms of the bile duct but not in exclusively intrahepatic cases. Mutational changes of these molecules appear therefore to be early alterations, because they are probably among the events initiating the intraductal epithelial proliferations. The further development of the intraductal proliferations to high-grade intraepithelial neoplasia and finally to invasive carcinoma was characterized by an increasing expression of nuclear TP53 and by the loss of SMAD4. The other genes remained either unchanged in their mutational status ( $p 16)$ or even showed a decreased frequency of mutations, as in the case of KRAS. This finding was unexpected and is difficult to interpret, as in pancreatic intraductal papillary mucinous neoplasm the frequency of KRAS mutations increases in parallel with the grade of malignancy. ${ }^{14}$ One possible explanation may be an insufficient number of microdissected tumor cells. Another reason might be that the progression to invasive carcinoma is based on the development of a new tumor cell clone distinct from that of the non-invasive cells and independent from KRAS activation.

The notion that KRAS and TP53 are important, at least at the beginning of the molecular pathogenesis of intraductal papillary neoplasm of the bile duct, is supported by a genetically engineered mouse model based on KRAS activation and TP53 deletion (AlbCre;Kras ${ }^{\mathrm{G} 12 \mathrm{D}} ; \mathrm{p5} 3^{\mathrm{L} / \mathrm{L}}$ and Alb-Cre;Kras $\left.{ }^{\mathrm{G} 12 \mathrm{D}} ; \mathrm{p} 53^{\mathrm{L} /}+\right)^{38}$ The animals with these genetic changes develop invasive intrahepatic cholangiocarcinoma from different precursor lesions, including intraductal papillary neoplasm of the bile duct-like tumors with intestinal and pancreato-biliary epithelium. ${ }^{38}$

Among the genes whose changes appear to be less important for the development of intraductal papillary neoplasms of the bile duct due to their infrequency are $G N A S$, a gene that was found to be linked to the molecular pathogenesis of pancreatic intraductal papillary mucinous neoplasm, ${ }^{14-16}$ $\beta$-catenin, HER2 and EGFR. Activating mutations of GNAS, which codes for a G-protein alphasubunit, were recently discovered in a high number of pancreatic intraductal papillary mucinous neoplasms, few invasive adenocarcinomas associated with intraductal papillary mucinous neoplasm and pancreatic intraepithelial neoplasia lesions and in a small number of intraductal papillary neoplasms of the bile duct. ${ }^{14-16,39}$ In our study, sequencing of exon 8 revealed a common 

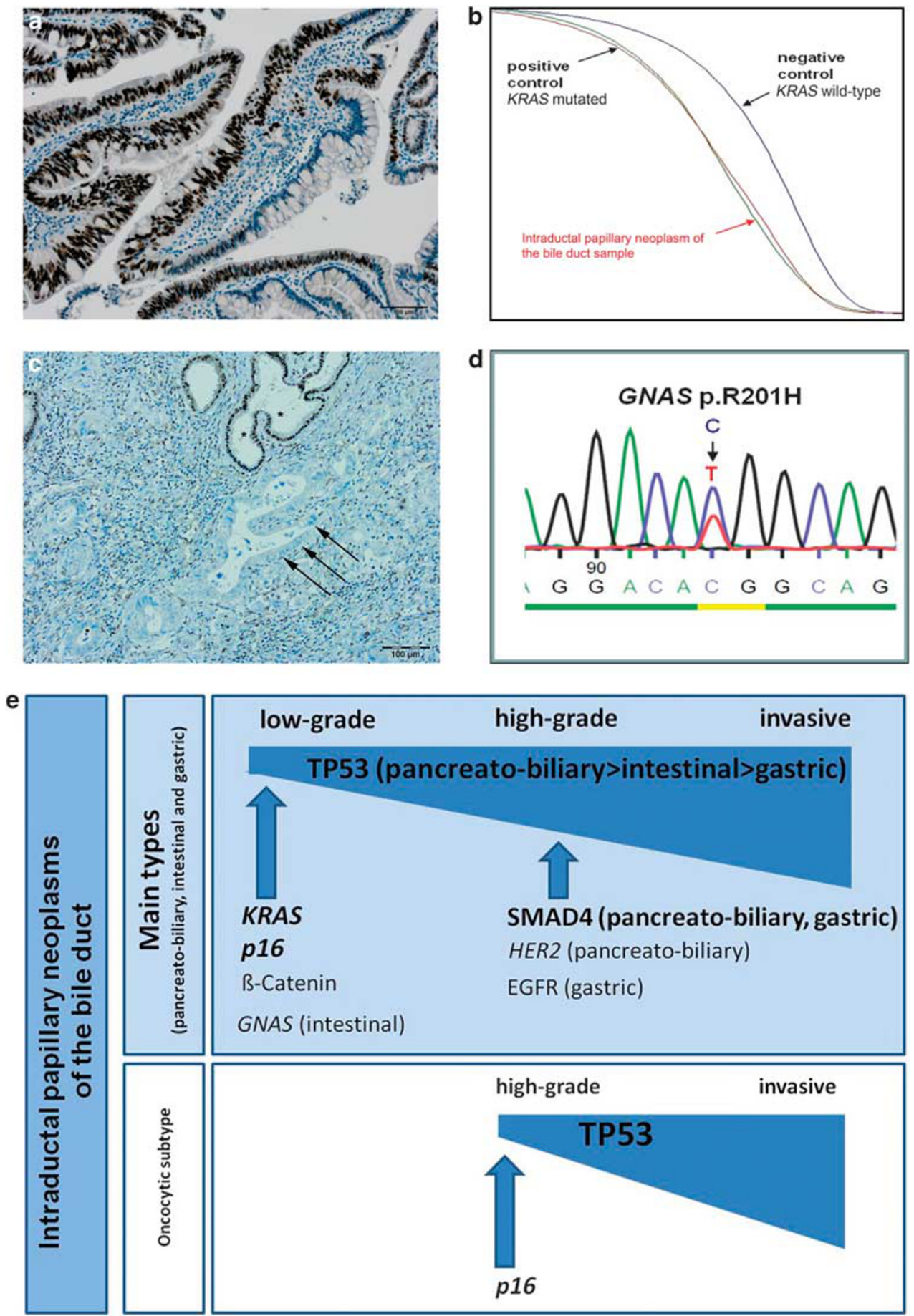

ß-Catenin GNAS (intestinal)

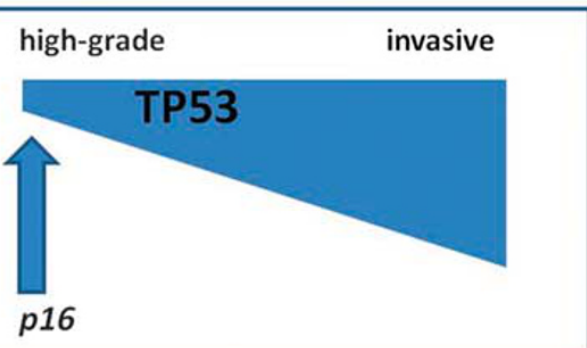

Figure 3 Oncogenic pathways in the carcinogenesis of intraductal papillary neoplasms of the bile duct. (a) Overexpression of TP53 was a common finding in non-invasive intraductal papillary neoplasms of the bile duct. (b) High-resolution melting analysis of KRAS (exon 2) revealed a mutation, which was confirmed by direct sequencing. (c) Loss of SMAD4 expression $(\rightarrow)$ in the invasive component of an intraductal papillary neoplasm of the bile duct with associated invasive carcinoma. Expression of SMAD4 in normal biliary glands served as internal control ( ${ }^{*}$ ). (d) Direct sequencing of GNAS discovered an activating mutation in exon 8 (p.R201H; reverse sequence) in an intraductal papillary neoplasm of the bile duct with low-grade intraepithelial neoplasia, intestinal subtype. (e) Proposed model of intraductal papillary neoplasm of the bile duct carcinogenesis. Analysis of genetic pathways in distinct intraductal papillary neoplasm of the bile duct subtypes revealed two distinct pathways of biliary intraductal papillary neoplasms: KRAS-dependent main type intraductal papillary neoplasms of the bile duct (pancreato-biliary, intestinal and gastric) and KRAS-independent oncocytic type intraductal papillary neoplasms of the bile duct.

activating mutation of GNAS (p.R201H) in one intraductal papillary neoplasms of the bile duct of the intestinal subtype and with low-grade intraepithelial neoplasia, whereas no GNAS mutations were detected in the other intraductal papillary neoplasms of the bile duct and in the 
cholangiocarcinomas. This low frequency is in accordance with data from a recent study, which included 23 intraductal papillary neoplasms of the bile duct and found only one GNAS-mutated case. ${ }^{39}$ Interestingly, the GNAS-mutated intraductal papillary neoplasms of the bile duct in the latter study $(1 / 23,4 \%)$ and our case as well as most of the GNAS-mutated pancreatic intraductal papillary mucinous neoplasms were all of the intestinal subtype. ${ }^{14,15,39}$ which may suggest a possible link at the molecular level to colorectal villous adenoma, where GNAS is commonly mutated (83\%). ${ }^{40}$

$\beta$-Catenin, a downstream target of the growthpromoting Wnt signalling pathway, was recently linked to progression of biliary intraductal papillary neoplasm of the bile duct associated with hepatolithiasis. $^{25}$ In our study, nuclear $\beta$-catenin accumulation was found only in four intraductal papillary neoplasms of the bile duct in non-invasive lesions. Similarly, rare were intraductal papillary neoplasms of the bile duct with amplified $(3+)$ HER2 or overexpressed EGFR. The occurrence of both receptors seems to be a late event in the progression of intraductal papillary neoplasms of the bile duct. As both growth receptors are druggable targets that have been successfully inhibited in different gastro-intestinal adenocarcinomas, they are also of potential interest for the treatment of advanced intraductal papillary neoplasms of the bile duct in the context of personalized medicine approaches. ${ }^{41}$ This may also hold true for cholangiocarcinoma, where EGFR was only positive in one case in contrast to that what has been reported in the literature. ${ }^{42,43}$ Using the established morphological and immunohistochemical criteria for subtype classification of pancreatic intraductal papillary mucinous neoplasms, ${ }^{6,31}$ we were able to classify the majority of intraductal papillary neoplasms of the bile duct (96\%) as pancreato-biliary, intestinal, gastric or oncocytic subtype. This indicates that, as expected, intraductal papillary neoplasms of the bile duct share many similarities with pancreatic intraductal papillary mucinous neoplasms from the histological point of view. However, some differences were noticed.

First of all, some differences were found concerning the relative frequency of the four subtypes. Pancreato-biliary (36\%) and intestinal (29\%) subtypes were the most common in the present series, followed by the gastric (18\%) and oncocytic (13\%) subtypes. The pancreato-biliary subtype was also most common in two recent studies, one from the United States $\left(69 \%{ }^{17}\right)$ and one from the Netherlands $\left(45 \%{ }^{18}\right)$. By contrast, the intestinal $\left(47 \%{ }^{23}\right.$ and $38 \%{ }^{21}$ ) subtype was the most frequent in Asian patients, which may be related to underlying intestinal metaplasia of the biliary epithelium driven by long-standing hepatolithiasis or Clonochiasis infection. The oncocytic subtype, on the other hand, seems to be rare in Asian cohorts $\left(6 \%^{21}\right.$ and $\left.3 \%^{23}\right)$, but more frequent in Western populations $\left(10 \%{ }^{18}\right.$ and $\left.15 \%{ }^{17}\right)$, including our study. Compared with the relative frequency of intraductal papillary mucinous neoplasm subtypes in the pancreas, where gastric and intestinal subtypes prevail, the gastric subtype appears much less common in intraductal papillary neoplasms of the bile duct in both the Eastern and Western cohorts.

Another difference between intraductal papillary neoplasms of the bile duct and pancreatic intraductal papillary mucinous neoplasms is the frequent aberrant expression of some of the mucin markers (eg, MUC1 in gastric and intestinal subtypes) and of the intestinal transcription factor CDX2 in all the subtypes. This fact points to transitions between the various subtypes and possibly to the existence of minor intraductal papillary neoplasm of the bile duct components with mixed differentiation. Moreover, two cases did neither fulfil the histological nor the immunohistochemical criteria of any of the subtypes and were therefore classified as not otherwise specified.

Concerning the molecular findings, the most obvious difference is the low incidence of KRAS and, in particular, GNAS mutations in intraductal papillary neoplasms of the bile duct compared with the pancreatic intraductal papillary mucinous neoplasms. ${ }^{14,39}$ This suggests differences in the molecular pathogenesis of the two entities, differences that might have a role in future treatment options. On the other hand, it is also of interest that the oncocytic subtype of intraductal papillary neoplasm of the bile duct showed no KRAS mutations, similar to what has been reported in pancreatic oncocytic subtypes, where KRAS mutations were less frequently detected compared with the other subtypes. ${ }^{44}$ Moreover, in both organ systems, oncocytic tumors had a better prognosis than other histological subtypes.

In pancreatic intraductal papillary mucinous neoplasms, the subtypes have a well-established prognostic relevance. ${ }^{6,9,10}$ This is unclear in intraductal papillary neoplasms of the bile duct. Kim et $a l^{23}$ reported longer survival for patients with gastric and intestinal subtypes compared with those with the pancreato-biliary subtype, where the frequency of invasive carcinoma was significantly higher $(73 \%)$ than those in the gastric and intestinal subtypes ( 27 and $33 \%$, respectively). ${ }^{23}$ Rocha et al ${ }^{17}$ were unable to find any survival differences between intraductal papillary neoplasm of the bile duct subtypes. We also observed no significant difference in the overall patient survival in the four subtypes, at least in part, because of the limited number of patients by subtype, although an invasive carcinoma was more frequently detected in the gastric (38\%) and pancreato-biliary subtypes (38\%) than the intestinal (23\%) and oncocytic (17\%) subtypes. When we compared the survival in patients with either intraductal papillary neoplasm or cholangiocarcinoma, patients with intraductal 
papillary neoplasms showed a slightly better, however, not statistically significant, overall survival than patients with cholangiocarcinoma. Whereas the 1-year survival rates of $93 \% \pm 5 \%$ in intraductal papillary neoplasm of the bile duct patients versus $81.3 \% \pm 9.8 \%$ in patients with cholangiocarcinoma point to a survival benefit of this distinct subgroup of biliary tumors, no major differences were observed in the 3-year survival rates (Table 1). To our surprise, no significant difference in survival was detected in subgroup comparison of patients with intraductal papillary neoplasm with invasive carcinoma versus patients without invasion (Figure 2). Follow-up revealed that at least a part of the patients $(2 / 20 ; 10 \%)$ who were histologically diagnosed with non-invasive intraductal papillary neoplasms of the bile duct developed invasive adenocarcinoma and died of the disease. Incomplete histological investigation of the lesions as well as multifocality and development of additional lesions within the biliary tract might explain the lack of significant difference in survival of patients with invasive and non-invasive intraductal papillary neoplasms of the bile duct. Longer observation in larger cohorts of patients and metaanalyses are necessary to further investigate whether patients with intraductal papillary neoplasm of the bile duct show survival benefit compared with patients with conventional cholangiocarcinoma.

Taken together, our data suggest that the development of intraductal papillary neoplasms of the bile duct follows an adenoma-carcinoma sequence, initiated by activation of KRAS and a loss of function of the tumor-suppressor genes TP53 and p16. Loss of SMAD4 takes place in late phases of tumor progression and might act as a driver to invasive carcinoma. Alterations of HER2, EGRF, $\beta$-catenin and GNAS appear to be less relevant in the carcinogenesis of intraductal papillary neoplasms of the bile duct. Alterations of the key genes and proteins in intraductal papillary neoplasm of the bile duct development, namely KRAS, TP53, p16 and SMAD4, were also commonly observed in cholangiocarcinomas. Reported mutation rates show a high range between intrahepatic and extrahepatic cholangiocarcinoma and between different studies. ${ }^{45}$ Intraductal papillary neoplasms of the bile duct and cholangiocarcinoma share, therefore, similarities in the respective molecular carcinogenesis but also display differences, ${ }^{28,45}$ as for example regarding the role of TP53 inactivation, which represents an early event in intraductal papillary neoplasms of the bile duct and a late event in cholangiocarcinoma development. ${ }^{29}$ Further evaluation of the molecular differences between cholangiocarcinomas originating from biliary intraepithelial neoplasia and intraductal papillary neoplasm of the bile duct might help to understand the distinct morphology and natural history of these two precursors of bile duct carcinoma.

\section{Disclosure/conflict of interest}

The authors declare no conflict of interest.

\section{References}

1 Zen Y, Adsay NV, Bardadin K, et al. Biliary intraepithelial neoplasia: an international interobserver agreement study and proposal for diagnostic criteria. Mod Pathol 2007;20:701-709.

2 Zen Y, Fujii T, Itatsu K, et al. Biliary papillary tumors share pathological features with intraductal papillary mucinous neoplasm of the pancreas. Hepatology 2006;44:1333-1343.

3 Albores-Saavedra J, Adsay NV, Crawford JM, et al. Carcinoma of the gallbladder and extrahepatic bile ducts, In: Bosman FT, Carneiro F, Hruban RH, Theise ND (eds) WHO Classification of Tumors of the Digestive System, 4th edn. IARC: Lyon, France; 2010, pp 266-273.

4 Nakanuma Y, Curado MP, Franceschi S, Gores G, Paradis V, Sripa B, Tsui WMS, Wee A. Intrahepatic cholangiocarcinoma, In: Bosman FT, Carneiro F, Hruban RH, Theise ND (eds) WHO Classification of Tumors of the Digestive System, 4th edn. IARC: Lyon, France, 2010.

5 Kloppel G, Kosmahl M. Is the intraductal papillary mucinous neoplasia of the biliary tract a counterpart of pancreatic papillary mucinous neoplasm? J Hepatol 2006;44:249-250.

6 Furukawa T, Kloppel G, Volkan Adsay N, et al. Classification of types of intraductal papillary-mucinous neoplasm of the pancreas: a consensus study. Virchows Arch 2005;447:794-799.

7 Furukawa T, Hatori T, Fujita I, et al. Prognostic relevance of morphological types of intraductal papillary mucinous neoplasms of the pancreas. Gut 2011;60:509-516.

8 Grutzmann R, Niedergethmann M, Pilarsky C, Kloppel $\mathrm{G}$, Saeger HD. Intraductal papillary mucinous tumors of the pancreas: biology, diagnosis, and treatment. Oncologist 2010;15:1294-1309.

9 Kim J, Jang KT, Mo Park S, et al. Prognostic relevance of pathologic subtypes and minimal invasion in intraductal papillary mucinous neoplasms of the pancreas. Tumour Biol 2011;32:535-542.

10 Takasu N, Kimura W, Moriya T, et al. Intraductal papillary-mucinous neoplasms of the gastric and intestinal types may have less malignant potential than the pancreatobiliary type. Pancreas 2010;39: 604-610.

11 Ohuchida K, Mizumoto K, Fujita $\mathrm{H}$, et al. Sonic hedgehog is an early developmental marker of intraductal papillary mucinous neoplasms: clinical implications of mRNA levels in pancreatic juice. J Pathol 2006;210:42-48.

12 Nissim S, Idos GE, Wu B. Genetic markers of malignant transformation in intraductal papillary mucinous neoplasm of the pancreas: a meta-analysis. Pancreas 2012;41:1195-1205.

13 Siveke JT, Einwachter H, Sipos B, et al. Concomitant pancreatic activation of $\operatorname{Kras}(\mathrm{G} 12 \mathrm{D})$ and Tgfa results in cystic papillary neoplasms reminiscent of human IPMN. Cancer Cell 2007;12:266-279. 
$14 \mathrm{Wu}$ J, Matthaei H, Maitra A, et al. Recurrent GNAS mutations define an unexpected pathway for pancreatic cyst development. Sci Transl Med 2011;3:92ra66.

15 Furukawa T, Kuboki Y, Tanji E, et al. Whole-exome sequencing uncovers frequent GNAS mutations in intraductal papillary mucinous neoplasms of the pancreas. Sci Rep 2011;1:161.

16 Kanda M, Matthaei $\mathrm{H}, \mathrm{Wu}$ J, et al. Presence of somatic mutations in most early-stage pancreatic intraepithelial neoplasia. Gastroenterology 2012;142(730.e9): 733.e9.

17 Rocha FG, Lee H, Katabi N, et al. Intraductal papillary neoplasm of the bile duct: A biliary equivalent to intraductal papillary mucinous neoplasm of the pancreas? Hepatology 2012;56:1352-1360.

18 Kloek JJ, van der Gaag NA, Erdogan D, et al. A comparative study of intraductal papillary neoplasia of the biliary tract and pancreas. Hum Pathol 2011;42:824-832.

19 Paik KY, Heo JS, Choi SH, Choi DW. Intraductal papillary neoplasm of the bile ducts: the clinical features and surgical outcome of 25 cases. J Surg Oncol 2008;97:508-512.

20 Yaman B, Nart D, Yilmaz F, et al. Biliary intraductal papillary mucinous neoplasia: three case reports. Virchows Arch 2009;454:589-594.

$21 \mathrm{Ji}$ Y, Fan J, Zhou J, et al. Intraductal papillary neoplasms of bile duct. A distinct entity like its counterpart in pancreas. Histol Histopathol 2008;23:41-50.

22 Choi SC, Lee JK, Jung JH, et al. The clinicopathological features of biliary intraductal papillary neoplasms according to the location of tumors. J Gastroenterol Hepatol 2010;25:725-730.

23 Kim KM, Lee JK, Shin JU, et al. Clinicopathologic features of intraductal papillary neoplasm of the bile duct according to histologic subtype. Am J Gastroenterol 2012;107:118-125.

24 Jung G, Park KM, Lee SS, et al. Long-term clinical outcome of the surgically resected intraductal papillary neoplasm of the bile duct. J Hepatol 2012;57: 787-793.

25 Itatsu K, Zen Y, Ohira S, et al. Immunohistochemical analysis of the progression of flat and papillary preneoplastic lesions in intrahepatic cholangiocarcinogenesis in hepatolithiasis. Liver Int 2007;27: 1174-1184.

26 Abraham SC, Lee JH, Boitnott JK, et al. Microsatellite instability in intraductal papillary neoplasms of the biliary tract. Mod Pathol 2002;15:1309-1317.

27 Abraham SC, Lee JH, Hruban RH, et al. Molecular and immunohistochemical analysis of intraductal papillary neoplasms of the biliary tract. Hum Pathol 2003;34:902-910.

28 Bickenbach K, Galka E, Roggin KK. Molecular mechanisms of cholangiocarcinogenesis: are biliary intraepithelial neoplasia and intraductal papillary neoplasms of the bile duct precursors to cholangiocarcinoma? Surg Oncol Clin N Am 2009;18:215-224; vii.

29 Nakanishi Y, Zen Y, Kondo S, et al. Expression of cell cycle-related molecules in biliary premalignant lesions: biliary intraepithelial neoplasia and biliary intraductal papillary neoplasm. Hum Pathol 2008;39:1153-1161.

30 Ludwig L, Buchler P, Kleeff J, et al. Multidisciplinary treatment of aggressive and rapidly progressing biliary papillomatosis. Dig Dis Sci 2010;55:3627-3629.

31 Asday NV, Fukushima N, Furukawa T, et al. Intraductal neoplasms of the pancreas, In: Bosman FT, Carneiro F, Hruban RH, Theise ND (eds) WHO Classification of Tumors of the Digestive System, 4th edn. IARC: Lyon, France; 2010, pp 304-313.

32 Hofmann M, Stoss O, Shi D, et al. Assessment of a HER2 scoring system for gastric cancer: results from a validation study. Histopathology 2008;52:797-805.

33 Valsecchi ME, McDonald M, Brody JR, et al. Epidermal growth factor receptor and insulinlike growth factor 1 receptor expression predict poor survival in pancreatic ductal adenocarcinoma. Cancer 2012;118:3484-3493.

34 Bettstetter M, Dechant S, Ruemmele P, et al. MethyQESD, a robust and fast method for quantitative methylation analyses in HNPCC diagnostics using formalin-fixed and paraffin-embedded tissue samples. Lab Invest 2008;88:1367-1375.

35 DJ S. Multiple comparison procedures: the practical solution. Am Statistician 1990;174-180.

36 Schutte M. DPC4/SMAD4 gene alterations in human cancer, and their functional implications. Ann Oncol 1999;10(Suppl 4):56-59.

37 Wilentz RE, Su GH, Dai JL, et al. Immunohistochemical labeling for dpc4 mirrors genetic status in pancreatic adenocarcinomas: a new marker of DPC4 inactivation. Am J Pathol 2000;156:37-43.

38 O'Dell MR, Huang JL, Whitney-Miller CL, et al. Kras(G12D) and p53 mutation cause primary intrahepatic cholangiocarcinoma. Cancer Res 2012;72: 1557-1567.

39 Matthaei H, Wu J, Dal Molin M, et al. GNAS codon 201 mutations are uncommon in intraductal papillary neoplasms of the bile duct. HPB (Oxford) 2012; 14:677-683.

40 Yamada M, Sekine S, Ogawa R, et al. Frequent activating GNAS mutations in villous adenoma of the colorectum. J Pathol 2012;228:113-118.

41 Geynisman DM, Catenacci DV. Toward personalized treatment of advanced biliary tract cancers. Discov Med 2012;14:41-57.

42 Yoshikawa D, Ojima H, Iwasaki M, et al. Clinicopathological and prognostic significance of EGFR, VEGF, and HER2 expression in cholangiocarcinoma. Br J Cancer 2008;98:418-425.

43 Shafizadeh N, Grenert JP, Sahai V, Kakar S. Epidermal growth factor receptor and HER-2/neu status by immunohistochemistry and fluorescence in situ hybridization in adenocarcinomas of the biliary tree and gallbladder. Hum Pathol 2010;41:485-492.

44 Xiao HD, Yamaguchi H, Dias-Santagata D, et al. Molecular characteristics and biological behaviours of the oncocytic and pancreatobiliary subtypes of intraductal papillary mucinous neoplasms. J Pathol 2011;224:508-516.

45 Hezel AF, Deshpande V, Zhu AX. Genetics of biliary tract cancers and emerging targeted therapies. J Clin Oncol 2010;28:3531-3540. 